\title{
Adulticidal Activity of Dillapiol and Semi-synthetic Derivatives of Dillapiol against Aedes aegypti (L.) (Culicidae)
}

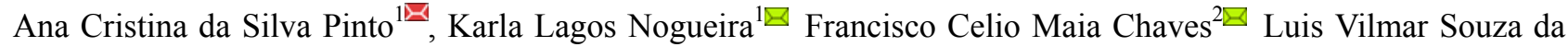
Silva $^{3}{ }^{3}$ Wanderli Pedro Tadei ${ }^{1 \Xi}$ Adrian Martin Pohlit ${ }^{4} \square$

1. Laboratory of Malaria and Dengue, Department of Society, Environment and Health, National Institute of Amazonian Research (INPA), Brazil

2. Embrapa Western Amazon; Vegetable and Medicinal Plant, Production Systems, AM Route 010, Km 29, 69010-970, Manaus, AM, Brazil

3. Laboratory of Water Resources, Department of Environmental Dynamics, INPA, Brazil

4. Laboratory of Active Principles of the Amazon (LAPAAM), Department of Technology and Innovation, INPA, Avenida Andre Araujo, 2936, 69060-001, Manaus, AM, Brazil

\Corresponding author email: ampohlit@gmail.com; $\square$ Authors

Journal of Mosquito Research, 2012, Vol.2, No.1 doi: 10.5376/jmr.2012.01.0001

Received: 15 Jun., 2012

Accepted: 04 Jul., 2012

Published: 28 Aus., 2012

This is an open access article published under the terms of the Creative Commons Attribution License, which permits unrestricted use, distribution, and reproduction in any medium, provided the original work is properly cited.

Preferred citation for this article:

Pinto et al., 2012, Adulticidal Activity of Dillapiol and Semi-synthetic Derivatives of Dillapiol against Aedes aegypti (L.) (Culicidae), Journal of Mosquito Research, Vol.2, No.1 1-7 (doi: 10.5376/jmr.2012.01.0001)

Abstract Phenylpropanoid dillapiol (1) isolated from the essential oil in leaves of Piper aduncum L. and thirteen semi-synthetic, $\mathrm{C}_{3}$ side-chain modified dillapiol derivatives 2-14 were evaluated for lethality against hemorrhagic dengue fever mosquito vector Aedes aegypti L.. First, adult female mosquitoes were exposed to these substances in a contact bioassay carried out in glass bottles at a single surface density $\left(0.57 \mu \mathrm{g} / \mathrm{cm}^{2}\right)$. Then, median lethal concentrations $\left(\mathrm{LC}_{50}\right)$ and $\mathrm{LC}_{90}$ were determined for active compounds. It was found that the structure of the $\mathrm{C}_{3}$ side chain was important for the toxic effects of these substances against $A$. aegypti adult females. Isodillapiol (2) and methyl, propyl and butyl 1-(2',3'-dimethoxy-4', $5^{\prime}$-methylenedioxyphenyl)propan-2-yl ethers (3, 5 and $\mathbf{6}$, respectively) all exhibited greater mosquitocidal activity than dillapiol (1). The order of molar per surface area of mosquitocidal activities was isodillapiol (2) $>$ butyl ether $\mathbf{6}>$ methyl ether $\mathbf{3}>$ propyl ether $\mathbf{5}>$ dillapiol (1). The potential of dillapiol and derivatives as $A$. aegypti mosquitocides was discussed.

Keywords Mosquitocidal activity; Isodillapiol; 2-methoxy dillapiol; 2-ethoxy dillapiol; 2-n-propoxy dillapiol; 2-n-butoxy dillapiol; Aedes aegypti

\section{Introduction}

The number of people afflicted by dengue hemorrhagic fever has risen in recent years. Aedes aegypti (L.) is the main vector of dengue hemorrhagic fever in the Americas. According to the World Health Organization (WHO, 2006), effective control of $A$. aegypti is attained with the use of chemical insecticides such as pyrethroids (cypermethrin, deltamethrin). However, contamination of the environments in the Amazon and other regions has occurred due to the use of pyrethroids and other long-lasting synthetic chemical insecticides for the control of Aedes spp.. Also, resistance of $A$. aegypti and other mosquito vectors to pyrethroids and other commercially available insecticides has increased in recent years as have the population densities of mosquitoes in many regions and urban centers (Campos and Andrade, 2001; Luna et al., 2004;
Barreto, 2005; Braga and Valle, 2007).

Bioactive chemicals isolated from plants are potential alternative agents for the control of $A$. aegypti and other insects (Chapagain et al., 2008). Research on the use of plant-derived chemicals to control mosquitoes and other insects has increased in recent years. This is especially true for the use of natural products based on plant essential oils (EOs) as insecticides and repellents (Shaalan et al., 2005; Georges et al., 2008; Nerio et al., 2010; Pohlit et al., 2011a; 2011b). It is firmly established that substances which contain a methylenedioxyphenyl nucleus such as synthetic piperonyl butoxide or components of EOs such as safrole, sesamolin, sesamin, myristicin and dillapiol (1) (Figure 1) have insecticide synergist and other properties which are important for insect control (Cassida, 1970; Alibhai, 1999). 


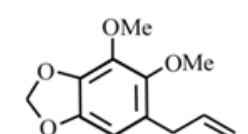

1

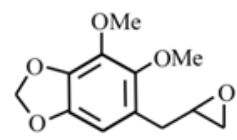

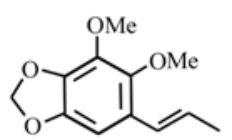

2

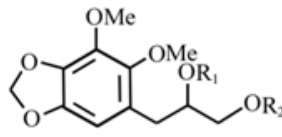

$9 \mathrm{R}_{1}=\mathrm{R}_{2}=\mathrm{H} ; 10 \mathrm{R}_{1}, \mathrm{R}_{2}=>\mathrm{C}\left(\mathrm{CH}_{3}\right)_{2}$

$11 \mathrm{R}_{1}=\mathrm{COCH}_{3}, \mathrm{R}_{2}=\mathrm{COCH}_{3}$

$12 \mathrm{R}_{1}, \mathrm{R}_{2}=>\mathrm{C}=\mathrm{O} ; 13 \mathrm{R}_{1}=\mathrm{H}, \mathrm{R}_{2}=\mathrm{CH}_{2} \mathrm{C}_{6} \mathrm{H}_{5}$

$14 \mathrm{R}_{1}=\mathrm{H}, \mathrm{R}_{2}=\mathrm{COC}_{6} \mathrm{H}_{5}$

Figure 1 Chemical structures of dillapiol (1) and semi-synthetic derivatives (2-14)

Dillapiol (1) is the major component of the EO obtained from the leaves of Piper aduncum L. (Cicció and Ballestero, 1997). It has insecticidal activity against Drosophila melanogaster ( $\left.\mathrm{LD}_{50} 0.27 \mathrm{mg} / \mathrm{jar}\right)$ and $A$. aegypti larvae (LD ${ }_{50} 11.0$ ppm, Lichtenstein et al., 1974). 1 is also known as an effective in vitro inhibitor of the epoxidase activity in larvae of the mosquito Ostrinia nubilalis (92\% inhibition). Epoxidase is important to insect defenses because it detoxifies insecticidal agents allowing mosquitoes and other insects to survive (Bernard et al., 1989; Demetzos et al., 2000). Also, 1 is an inhibitor of cytochrome polymerase- $\beta \mathrm{P} 450$ and it inhibits the activity of the polysubstrate monooxygenases which are used by insects to metabolize toxins as well as enzyme-lyase activity (Larocque et al., 1999; Amiguet et al., 2006).

Dillapiol (1) exhibits synergism with several classes of substances used in insect control. For example, synergism of $\mathbf{1}$ was observed with pyrethrins (factor of synergism 2.0) against the wheat flour beetle Tribolium castaneum (Tomar et al., 1979a; 1979b) with $\alpha$-terthienyl against larvae of Aedes atropalpus $\left(\mathrm{LC}_{50} 41.1 \mathrm{ng} / \mathrm{mL}\right)$ and factor of synergism 1.9 (Majerus, 1997; Alibhai, 1999; Belzile et al., 2000) with pyrethrin and carbaryl against adults of the red flour beetle Tribolium castaneum (Larocque et al., 1999). Also, 1 exhibits synergism with the pyrethroids deltamethrin and cypermethrin providing 70\% and $100 \%$ mortality in adult $A$. aegypti in $90 \mathrm{~min}$ and $15 \mathrm{~min}$, respectively. Furthermore, combination of $\mathbf{1}$ with $\alpha$-cypermethrin led to $100 \%$ mortality against Anopheles albitarsis adults in $45 \mathrm{~min}$ (Gomes et al., 2005; 2006). 1 exhibits antifungal synergistic effects in combination with the sesquiterpene dialdehyde cinnamodial against Alternaria alternata, Candida albicans and Wangiella dermatitides (Amiguet et al., 2006). Also, 1 exhibits synergistic activity in combination with gedunin $(79.0 \pm 5.3) \%$ as an active antimalarial (Omar et al., 2003).

Semi-synthetic derivatives of $\mathbf{1}$ which exhibit intact methylenedioxyphenyl nuclei also exhibit synergism against insect species. For example, derivatives 2-6 (Figure 1) and other derivatives of $\mathbf{1}$ exhibited in vitro synergism (2.8 5.0 fold increased effects) with pyrethrin insecticides against the wheat flour beetle Tribolium castaneum (Tomar et al., 1979a; 1979b; Mukerjee et al., 1979) and with $\alpha$-tertienyl in larvae of Aedes atropalpus where derivative 5 showed synergism as evidenced by 1.5 fold increased activity (Majerus, 1997; Belzile, 2000).

The general aim of this work was to identify semi-synthetic derivatives of $\mathbf{1}$ exhibiting adulticidal activity against Aedes aegypti found in the Brazilian Amazon. Thus, known dillapiol derivatives 2-6 and 9 and new derivatives 7-8 and 10-14 were prepared herein and evaluated for contact adulticidal activity against the hemorrhagic dengue fever vector Aedes aegypti. To our knowledge, this is the first time that $\mathbf{1}$ and derivatives 2-14 have been evaluated for adulticidal activity against mosquitoes.

\section{Results and Discussion}

Initially, adulticidal activity of dillapiol (1) and derivatives 2-14 was determined against $A$. aegypti adult females at a surface density of $0.57 \mu \mathrm{g} / \mathrm{cm}^{2}$. Dillapiol (1) and isodillapiol (2) were the most active substances (100\% mortality after exposure for $45 \mathrm{~min}$ ) followed by methyl, ethyl, propyl and butyl 1-(2',3'-dimethoxy-4',5'-methylenedioxyphenyl)propan-2-yl ethers 3-6 which exhibited lethalities of $80 \% \sim 98 \%$ to A. aegypti adult females after an exposure time of 90 min. Dillapiol oxide (8) killed about half (51\%) and acetonide 10 killed $29 \%$ of mosquitoes after 90 min of exposure. Other derivatives of dillapiol 
(11-14) were not effective (4-11\% mortality) at killing A. aegypti adult females (Figure 2). Thus, dillapiol derivatives 8-14 exhibited lower adulticide potency than dillapiol (1) or isodillapiol (2) against A. aegypti. Taken together, this initial result demonstrated that functionalization of both the 2- and 3-positions on the propyl side chains with oxygen atoms or oxygencontaining moieties was associated with decreased adulticide activity compared to $\mathbf{1}$ or $\mathbf{2}$.

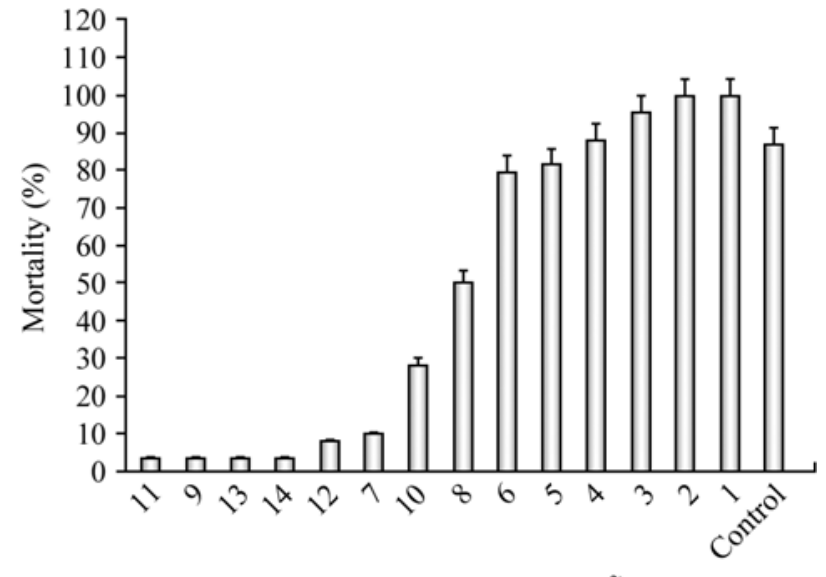

Compounds $\left(0.57 \mu \mathrm{g} / \mathrm{cm}^{2}\right)$

Figure 2 Percentage mortalities for dillapiol and derivatives against Aedes aegypti L. adult females after exposure for 90 mins to $0.57 \mu \mathrm{g} / \mathrm{cm}^{2}$ of sample or control substance $\alpha$-cypermethrin $\left(0.59 \mathrm{ng} / \mathrm{cm}^{2}\right)$.

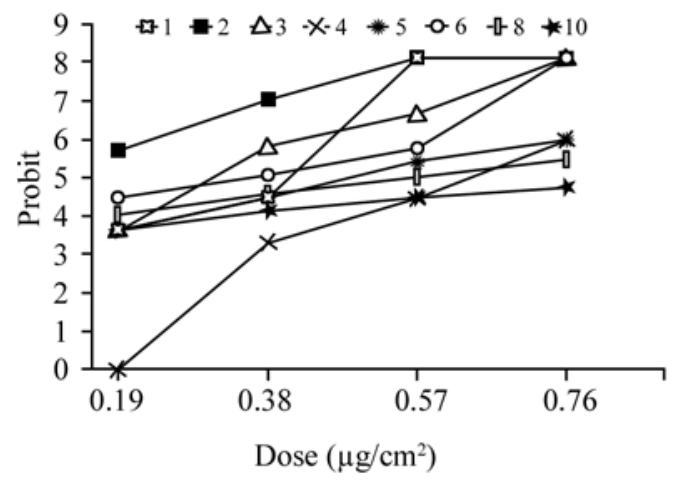

Figure 3 Probit analysis of the mortality caused by dillapiol and its derivatives against Aedes aegypti female adults (90 min exposure).

The more active substances found in the initial screening described above were then evaluated to establish the relationship between concentration and adulticidal activity in $A$. aegypti. The results of probit analysis are shown in Figure 3. The adulticide activity of ethyl ether $\mathbf{4}$ exhibited the greatest dependence on surface density in the range of surface densities evaluated and disappeared altogether at the lowest density tested $\left(0.19 \mu \mathrm{g} / \mathrm{cm}^{2}\right)$.

Median lethal concentration $\left(\mathrm{LC}_{50}\right)$ and $\mathrm{LC}_{90}$ and other statistical parameters for dillapiol and derivatives against $A$. aegypti adult females are presented in Table 1. Interestingly, the most active mosquitocide was a structural isomer of dillapiol (1), isodillapiol (2), which was 3-fold more active than $\mathbf{1}$. Also, several alkyl 1-(2',3'-dimethoxy-4',5'methylenedioxyphenyl)propan-2-yl ethers exhibited significant adulticide activity. Given the inactivity of octyl ether $\mathbf{7}$ and the adulticidal activities of methyl, ethyl, propyl and butyl ethers 3-6, there appeared to be an optimal alkyl chain length. On a molar basis, the order of decreasing contact adulticide activity was: isodillapiol (2) > butyl ether $6>$ methyl ether $3>$ propyl ether $\mathbf{5}>$ dillapiol (1) $>$ epoxide $8>$ ethyl ether $4>$ acetonide $\mathbf{1 0}$.

Results on the mortality of $A$. aegypti adult females reported in the present study confirmed the potential of dillapiol, isodillapiol and semi-synthetic ether derivatives as mosquitocides. Furthermore, varying the chain length/structure of the alkyl group attached to the dillapiol carbon skeleton in ether derivatives could be a means to modulate physical and chemical properties such as solubility and volatility leading to improved utility and mosquitocidal activity of these derivatives in field situations.

\section{Methods}

\subsection{Isolation of 1}

Isolation of $\mathbf{1}$ from Piper aduncum has been described previously (Pinto, 2008; Pohlit et al., 2008). Briefly, EO of $P$. aduncum growing in the region near Manaus, Amazonas State, Brazil was prepared on a pilot scale $(600 \mathrm{~mL}, \mathrm{~d}=1.082 \mathrm{~g} / \mathrm{mL}, 2.4 \%$ based on dry weight of plant) by steam distillation of sun-dried leaves. Then, $1(17.7 \mathrm{~g}, 73.4 \% \mathrm{w} / \mathrm{w}$ based on EO and $1.70 \% \mathrm{w} / \mathrm{w}$ based on dry plant) was obtained by fractional vacuum distillation of EO (dillapiol fraction collected at $\left.4 \mathrm{cmHg}, c a \cdot 157^{\circ} \mathrm{C}\right)$. 
Table 1 Adulticidal activities of dillapiol (1) and derivatives of dillapiol against Aedes aegypti adult females after 90 mins of exposure.

\begin{tabular}{|c|c|c|c|c|c|c|c|}
\hline No. & $\begin{array}{l}\mathrm{LC}_{50}(\mathrm{CI}) \\
\mu \mathrm{g} / \mathrm{cm}^{2}\end{array}$ & $\begin{array}{l}\mathrm{LC}_{90}(\mathrm{CI}) \\
\mu \mathrm{g} / \mathrm{cm}^{2}\end{array}$ & $\begin{array}{l}\text { Slope } \\
\pm 100\end{array}$ & SD & $\begin{array}{l}\text { Chi-square } \\
\chi^{2}\end{array}$ & $\begin{array}{l}\mathrm{LC}_{50}(\mathrm{CI}) \\
\mathrm{nmol} / \mathrm{cm}^{2}\end{array}$ & $\begin{array}{l}\mathrm{LC}_{90}(\mathrm{CI}) \\
\mathrm{nmol} / \mathrm{cm}^{2}\end{array}$ \\
\hline 1 & $\begin{array}{l}0.381 \\
(0.319 \sim 0.442)\end{array}$ & $\begin{array}{l}0.575 \\
(0.514 \sim 0.636)\end{array}$ & $7.2 \pm 0.7$ & 0.3 & 32.1 & $\begin{array}{l}1.72 \\
(1.437-1.991)\end{array}$ & $\begin{array}{l}2.59 \\
(1.085-1.459)\end{array}$ \\
\hline 2 & $\begin{array}{l}0.136 \\
(0.087 \sim 0.167)\end{array}$ & $\begin{array}{l}0.274 \\
(0.241 \sim 0.324)\end{array}$ & $4.2 \pm 1.0$ & 0.6 & 1.3 & $\begin{array}{l}0.61 \\
(0.391-0.752)\end{array}$ & $\begin{array}{l}1.23 \\
(1.09-1.46)\end{array}$ \\
\hline 3 & $\begin{array}{l}0.295 \\
(0.264 \sim 0.326)\end{array}$ & $\begin{array}{l}0.457 \\
(0.409 \sim 0.531)\end{array}$ & $6.8 \pm 0.8$ & 0.4 & 0.9 & $\begin{array}{l}1.16 \\
(1.04-1.28)\end{array}$ & $\begin{array}{l}1.80 \\
(1.61-2.09)\end{array}$ \\
\hline 4 & $\begin{array}{l}0.618 \\
(0.546 \sim 0.709)\end{array}$ & $\begin{array}{l}0.845 \\
(0.729 \sim 1.234)\end{array}$ & $9.4 \pm 1.4$ & 0.3 & 2.7 & $\begin{array}{l}2.31 \\
(2.04-2.65)\end{array}$ & $\begin{array}{l}3.15 \\
(2.72-4.60)\end{array}$ \\
\hline 5 & $\begin{array}{l}0.438 \\
(0.316 \sim 0.604)\end{array}$ & $\begin{array}{l}1.065 \\
(0.723 \sim 3.555)\end{array}$ & $3.3 \pm 0.4$ & 0.2 & 3.3 & $\begin{array}{l}1.55 \\
(1.12-2.14)\end{array}$ & $\begin{array}{l}3.78 \\
(2.56-12.60)\end{array}$ \\
\hline 6 & $\begin{array}{l}0.315 \\
(0.254 \sim 0.377)\end{array}$ & $\begin{array}{l}0.699 \\
(0.638 \sim 0.761)\end{array}$ & $3.7 \pm 0.5$ & 0.2 & 7.8 & $\begin{array}{l}1.06 \\
(0.857-1.27)\end{array}$ & $\begin{array}{l}2.36 \\
(2.153-2.56)\end{array}$ \\
\hline 8 & $\begin{array}{l}0.531 \\
(0.443 \sim 0.635)\end{array}$ & $\begin{array}{l}1.855 \\
(1.269 \sim 3.835)\end{array}$ & $2.3 \pm 0.5$ & 0.2 & 1.0 & $\begin{array}{l}2.23 \\
(1.86-2.67)\end{array}$ & $\begin{array}{l}7.79 \\
(5.33-16.11)\end{array}$ \\
\hline 10 & $\begin{array}{l}1.177 \\
(0.822 \sim 3.038)\end{array}$ & $\begin{array}{l}6.600 \\
(2.703 \sim 88.52)\end{array}$ & $1.7 \pm 0.5$ & 0.2 & 0.01 & $\begin{array}{l}3.98 \\
(2.78-10.26)\end{array}$ & $\begin{array}{l}22.30 \\
(9.13-29.90)\end{array}$ \\
\hline${ }^{*}$ Ctrl & $\begin{array}{l}0.494 * * \\
(0.443 \sim 0.549)\end{array}$ & $\begin{array}{l}0.876^{* *} \\
(0.761 \sim 1.069)\end{array}$ & $5.2 \pm 0.7$ & 0.2 & 2.9 & $\begin{array}{l}1.19 * * * \\
(1.06 \sim 1.31)\end{array}$ & $\begin{array}{l}2.10 * * * \\
(1.83 \sim 2.57)\end{array}$ \\
\hline
\end{tabular}

Note: $\mathrm{LC}_{50}=$ Lethal concentration at which $50 \%$ of mosquitoes were killed. $\mathrm{LC}_{90}=$ Lethal concentration at which $90 \%$ of mosquitoes were killed. $\mathrm{CL}=$ confidence limits at $90 \%$ probability. ${ }^{*} \mathrm{Control}=\alpha$-cypermethrin, $* * \mathrm{ng} / \mathrm{cm}^{2}, * *$ pmol $/ \mathrm{cm}^{2}$. Values are the mean of four replicates with $\pm(\mathrm{SEM}))$ standard error.

\subsection{Preparation of derivatives of 1}

Isomerization, oxymercuration, epoxidation, bishydroxylation (oxidation) and reactions using $\mathbf{1}$ as substrate yielded: isodillapiol (2), methyl, ethyl, propyl, butyl and octyl 1,2-dihydrodillapiol-2-yl ethers 3-7, dillapiol epoxide (8) and 1,2-dihydroxy-1,2-dihydrodillapiol (9). Acetonide formation, carbonylation, diacetylation, benzylation and benzoylation of the hydroxyl groups in $\mathbf{9}$ provided: 1,2-dihydroxy-1,2-dihydrodillapiol acetonide $\mathbf{1 0}$ and carbonate 12, $O, O$-diacetyl, 1-O-benzyl and 1-O-benzoyl derivatives of 1,2-dihydroxy-1,2dihydrodillapiol 11, 13 and 14, respectively (Scheme 1) as described in Pinto (2008) and Pohlit et al. (2008).

\subsection{Adulticidal activity of semi-synthetic substances against $A$. aegypti}

$A$. aegypti were maintained in the insectary of the INPA Department of Society, Environment and Health in alternating light and dark photoperiods of $12 \mathrm{~h}$. They were fed periodically with the blood of rats as a source of protein used mainly for the production of eggs.

The acute activity of the above semi-synthetic derivatives was determined by contact with $A$. aegypti adult females. Substances were dissolved in HPLC grade acetone at a concentration of $10 \mathrm{mg} / \mathrm{mL}$. Volumes of this sample solution were measured using an adjustable pipette and transferred to clear glass bottles. Sample was homogenized in each bottle and complete evaporation of the solvent was accomplished by leaving bottles open to the air overnight. The same procedure was performed for both positive controls ( $\alpha$-cypermethrin at a concentration of $0.59 \mathrm{ng} / \mathrm{cm}^{2}$ in acetone) and negative controls (1.0 mL of acetone). Then, 15 female hematophagous $A$. aegypti aged 3 to 5 days were placed in each bottle at $25^{\circ} \mathrm{C} \sim 27^{\circ} \mathrm{C}$ and $50 \% \sim 70 \%$ humidity and controlled according to $\mathrm{WHO}$ recommendations (WHO, 2006). The bioassay was performed in triplicate making observations every $15 \mathrm{~min}$ until exposure time to substances reached $90 \mathrm{~min}$. 
Mortality was defined as immobility and movement of mosquitoes was recorded even if immobilized mosquitoes started recovering $2 \mathrm{~h}$ after treatment. There was no mortality in negative control bottles.

\subsection{Statistical analysis}

Data on the mortality of mosquitoes (immobility, absence of movement) were analyzed using the program StatsDirect 2.6.6 (Stats Direct Limited).

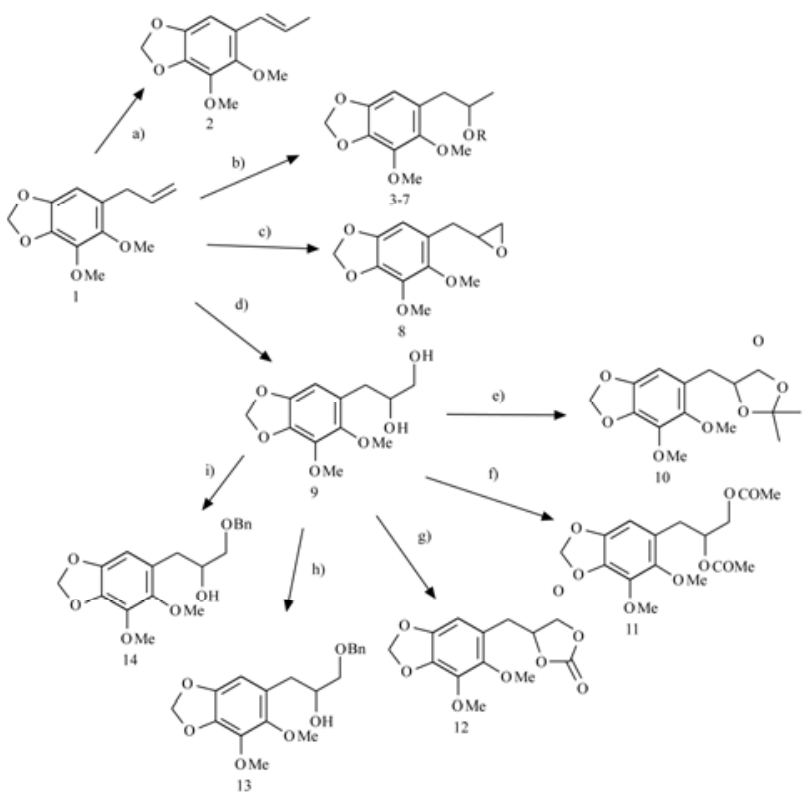

Scheme 1 Synthesis of dillapiol derivatives. Reagents and conditions: a) $\mathrm{KOH} / \mathrm{EtOH} 17 \%$, reflux, $24 \mathrm{~h}, 81 \%$; b) $\mathrm{THF} / \mathrm{Hg}(\mathrm{OH})_{2}$, alcohol, $\mathrm{NaBH}_{4} / \mathrm{KOH}, \mathrm{rt}, 3: \mathrm{R}=\mathrm{Me}, 78 \%$, 4: $\mathrm{R}=\mathrm{Et}, 62 \%, 5: \mathrm{R}=\mathrm{Pr}, 77 \%, \mathbf{6}: \mathrm{R}=\mathrm{But}, 42 \%, 7: \mathrm{R}=$ Oct, $17 \%$; c) $m$-CPBA, $\mathrm{CH}_{2} \mathrm{Cl}_{2},-5{ }^{\circ} \mathrm{C}(2 \mathrm{~h}), \mathrm{rt}, 240 \mathrm{~h}, 11 \%$; d) acetone, $\mathrm{KMnO}_{4}$, rt, 24h, 63\%; e) $\left(\mathrm{CH}_{3}\right)_{2} \mathrm{C}\left(\mathrm{OCH}_{3}\right)_{2}, p-\mathrm{CH}_{3} \mathrm{C}_{6} \mathrm{H}_{4} \mathrm{SO}_{3} \mathrm{H}, \mathrm{rt}$, 120 h, 82\%; (6) $\left(\mathrm{CH}_{3} \mathrm{CO}\right)_{2} \mathrm{O}$, pyridine, rt, $24 \mathrm{~h}, 58 \%$; (7) $\mathrm{CO}\left(\mathrm{OCCl}_{3}\right)_{2}, \mathrm{CH}_{2} \mathrm{Cl}_{2}, \mathrm{Et}_{3} \mathrm{~N}, 0{ }^{\circ} \mathrm{C}$, rt, 39\%; (8) $\mathrm{C}_{6} \mathrm{H}_{5} \mathrm{Br}, \mathrm{DMF}$, $\mathrm{K}_{2} \mathrm{CO}_{3}, 0{ }^{\circ} \mathrm{C}$, rt, $12 \%$; (9) $\mathrm{C}_{6} \mathrm{H}_{5} \mathrm{CH}_{2} \mathrm{Cl}$, pyridine, rt, $32 \%$.

Significant differences were determined among treatments at 0.05 space level of significance. The average values of the absolute frequencies of mortality in those treatments with a dosage of $150 \mu \mathrm{g}$ were analyzed for variance with free Kruskal-Wallis distribution $(p<0.05)$. Then, these data underwent a test of medium contrast at $5 \%$ level of significance using the procedure of Conover-Inman (Table 1, Conover, 1999). Data were evaluated by probit analysis (POLOPC program LeOra Software 1987) to determine the $\mathrm{LC}_{50}$ and $\mathrm{LC}_{90}$ values (in $\mu \mathrm{g} / \mathrm{cm}^{2}$ ) that caused $50 \%$ and $90 \%$ mortalities with $95 \%$ confidence intervals.

\section{Conclusion}

Isodillapiol and methyl, propyl and butyl 1,2-dihydrodillapiol-2-yl ethers were all more lethal than the isolated phenylpropanoid dillapiol to Aedes aegypti adult females, and they all have potential as contact mosquitocides. Remembering that the important insecticide synergist piperonyl butoxide is also a phenylpropanoid derivative, dillapiol derivatives will be further investigated for synergism with commercial pyrethroid products used presently in the Amazon and other regions of the world for mosquito vector control.

\section{Experimental}

${ }^{1} \mathrm{H}$ and ${ }^{13} \mathrm{C}$ NMR spectra were collected at $500 \mathrm{MHz}$ and $125 \mathrm{MHz}$, respectively. The derivatives have the following spectral data:

7: Colorless oil. ${ }^{1} \mathrm{H}$ NMR $\left(\mathrm{CDCl}_{3}\right): \delta 6.39(s, 1 \mathrm{H})$, $5.88(s, 2 \mathrm{H}), 4.01(s, 3 \mathrm{H}), 3.76(s, 3 \mathrm{H}), 3.55$ (sext, $J=6.0 \mathrm{~Hz}, 1 \mathrm{H}), 3.46(d t, J=6.5,9.3 \mathrm{~Hz}, 1 \mathrm{H}), 3.36(d t$, $\mathrm{J}=6.5,9.3 \mathrm{~Hz}, 1 \mathrm{H}), 2.82(d d, J=6.0,13.5 \mathrm{~Hz}, 1 \mathrm{H})$, $2.52(d d, J=7.0,13.5 \mathrm{~Hz}, 1 \mathrm{H}), 1.51(m, 2 \mathrm{H}), 1.26(m$, $10 \mathrm{H}), 1.11(d, J=6.0 \mathrm{~Hz}, 3 \mathrm{H}), 0.88(t, J=6.7, \mathrm{~Hz}, 3 \mathrm{H})$ ppm; ${ }^{13} \mathrm{C}$ NMR/DEPT $\left(\mathrm{CDCl}_{3}\right): \delta$ 144.9(C); 144.5(C); 137.7(C); 136.1(C); 125.5(C); 103.9(CH); 101.2( $\left(\mathrm{CH}_{2}\right)$; $76.2(\mathrm{CH}) ; \quad 69.0\left(\mathrm{CH}_{2}\right) ; \quad 61.3\left(\mathrm{CH}_{3}\right) ; \quad 60.1\left(\mathrm{CH}_{3}\right) ;$ $37.2\left(\mathrm{CH}_{2}\right) ; \quad 32.1\left(\mathrm{CH}_{2}\right) ; \quad 30.3\left(\mathrm{CH}_{2}\right) ; \quad 29.7\left(\mathrm{CH}_{2}\right)$; $29.5\left(\mathrm{CH}_{2}\right) ; \quad 26.4\left(\mathrm{CH}_{2}\right) ; \quad 22.9\left(\mathrm{CH}_{2}\right) ; \quad 19.9\left(\mathrm{CH}_{3}\right)$; 14.3 $\left(\mathrm{CH}_{3}\right)$ ppm. EI-MS: $m / z 352[\mathrm{M}+\cdot]$.

8: Yellow oil. ${ }^{1} \mathrm{H}$ NMR $\left(\mathrm{CDCl}_{3}\right): \delta 6.43(s, 1 \mathrm{H}), 5.89$ $(s, 2 \mathrm{H}), 4.02(s, 3 \mathrm{H}), 3.77(s, 3 \mathrm{H}), 3.09(m, 1 \mathrm{H}), 2.78$ $(m, 3 \mathrm{H}), 2.55(d d, J=2.6,5.2 \mathrm{~Hz}, 1 \mathrm{H}) \mathrm{ppm}$. EI-MS: $\mathrm{m} / \mathrm{z} 238[\mathrm{M}+\cdot]$.

9: White solid. ${ }^{1} \mathrm{H}$ NMR $\left(\mathrm{CDCl}_{3}\right): \delta 6.37(s, 1 \mathrm{H}), 5.90$ $(s, 2 \mathrm{H}), 4.02(s, 3 \mathrm{H}), 3.84(m, 1 \mathrm{H}), 3.59(d d, J=3.7$, $11.1 \mathrm{~Hz}, 1 \mathrm{H}), 3.47(d d, J=6.0,11.1 \mathrm{~Hz}, 1 \mathrm{H}), 3.79(s$, $3 \mathrm{H}), 2.73(d, J=6.5 \mathrm{~Hz}, 2 \mathrm{H}), 2.18(s, 2 \mathrm{H}) \mathrm{ppm} ;{ }^{13} \mathrm{C}$ NMR/DEPT $\left(\mathrm{CDCl}_{3}\right): \delta 144.7(\mathrm{C}), 144.4(\mathrm{C}), 137.6(\mathrm{C})$, 136.3(C), 103.4(CH), 123.5(C), 101.2( $\left.\mathrm{CH}_{2}\right), 72.7(\mathrm{CH})$, 65.7 $\left(\mathrm{CH}_{2}\right), 61.2\left(\mathrm{CH}_{3}\right)$, 59.9 $\left(\mathrm{CH}_{3}\right), 34.1\left(\mathrm{CH}_{2}\right)$ ppm. EI-MS: $m / z 256[\mathrm{M}+\cdot]$. 
10: Yellow oil. ${ }^{1} \mathrm{H}$ NMR $\left(\mathrm{CDCl}_{3}\right): \delta 5.91(d, J=1.5 \mathrm{~Hz}$, $1 \mathrm{H}), 5.88(d, J=1.5 \mathrm{~Hz}, 2 \mathrm{H}), 3.99(s, 3 \mathrm{H}), 3.84(\mathrm{~m}$, $1 \mathrm{H}), 3.77(d d, J=3.0,11.3 \mathrm{~Hz}, 1 \mathrm{H}), 3.75(s, 3 \mathrm{H}), 3.66$ $(d d, J=7.0,11.3 \mathrm{~Hz}, 1 \mathrm{H}) ; 2.67(d d, J=2.8,16.3 \mathrm{~Hz}$, $1 \mathrm{H}), 2.38(d d, J=11.0,16.3 \mathrm{~Hz}, 1 \mathrm{H}), 1.56(s, 3 \mathrm{H}), 1.51$ $(s, 3 \mathrm{H}) . \mathrm{ppm} ;{ }^{13} \mathrm{C}$ NMR/DEPT $\left(\mathrm{CDCl}_{3}\right): \delta 143.9(\mathrm{C})$, 140.1(C), 136.3(C), 136.2(C), 119.9(C), 119.3(C), $101.1\left(\mathrm{CH}_{2}\right), \quad 69.6(\mathrm{CH}), \quad 66.1\left(\mathrm{CH}_{2}\right), \quad 60.9\left(\mathrm{CH}_{3}\right)$, $60.3\left(\mathrm{CH}_{3}\right), \quad 73.8(\mathrm{CH}), \quad 28.7\left(\mathrm{CH}_{3}\right), \quad 25.8\left(\mathrm{CH}_{3}\right)$, 25.1( $\left(\mathrm{CH}_{2}\right) \mathrm{ppm}$. EI-MS: $\mathrm{m} / z 296[\mathrm{M}+\cdot]$.

11: Yellow oil. ${ }^{1} \mathrm{H}$ NMR $\left(\mathrm{CDCl}_{3}\right): \delta 6.34(s, 1 \mathrm{H}), 5.89$ $(s, 2 \mathrm{H}), 5.24$ (dddd, $J=3.3,3 \times 6.6 \mathrm{~Hz}, 1 \mathrm{H}), 4.23(d d$, $J=3.3,12.0 \mathrm{~Hz}, 1 \mathrm{H}), 4.02(d d, J=6.6,12.0 \mathrm{~Hz}, 1 \mathrm{H})$, $4.00(s, 3 \mathrm{H}), 3.79(s, 3 \mathrm{H}), 2.86(d d, J=6.6,14.0 \mathrm{~Hz}$, $1 \mathrm{H}), 2.78(d d, J=6.6,14.0 \mathrm{~Hz}, 1 \mathrm{H}), 2.06(s, 3 \mathrm{H}), 2.03$ $(s, 3 \mathrm{H}) \mathrm{ppm} ;{ }^{13} \mathrm{C}$ NMR/DEPT $\left(\mathrm{CDCl}_{3}\right): \delta 170.9(\mathrm{C})$, 170.6(C), 145.3(C), 144.7(C), 137.8(C), 136.7(C), $122.3(\mathrm{C}), \quad 103.6(\mathrm{CH}), \quad 101.5\left(\mathrm{CH}_{2}\right), \quad 71.9(\mathrm{CH})$, $64.8\left(\mathrm{CH}_{2}\right), \quad 61.4\left(\mathrm{CH}_{3}\right), \quad 60.2\left(\mathrm{CH}_{3}\right), \quad 31.4\left(\mathrm{CH}_{2}\right)$, 21.3 $\left(\mathrm{CH}_{3}\right), 21.0\left(\mathrm{CH}_{3}\right)$ ppm. EI-MS: $m / z 340[\mathrm{M}+\cdot]$.

12: Yellow oil. ${ }^{1} \mathrm{H}$ NMR $\left(\mathrm{CDCl}_{3}\right): \delta 6.36(s, 1 \mathrm{H}), 5.91$ $(s, 2 \mathrm{H}), 4.89(\mathrm{~m}, 1 \mathrm{H}), 4.40(t, J=8.6 \mathrm{~Hz}, 1 \mathrm{H}), 4.18(d d$, $J=7.0,8.6 \mathrm{~Hz}, 1 \mathrm{H}), 4.03(s, 3 \mathrm{H}), 3.78(s, 3 \mathrm{H}), 3.05(d d$, $J=6.0,14.0 \mathrm{~Hz}, 1 \mathrm{H}), 2.89(d d, J=7.0,14.0 \mathrm{~Hz}, 1 \mathrm{H})$ $\mathrm{ppm} ;{ }^{13} \mathrm{C} \mathrm{NMR} / \mathrm{DEPT}\left(\mathrm{CDCl}_{3}\right): \delta$ 155.1(C); 145.2(C); $145.0(\mathrm{C}) ; 137.9(\mathrm{C}) ; 137.3(\mathrm{C}) ; 119.7(\mathrm{C}) ; 103.6(\mathrm{CH})$; $101.7\left(\mathrm{CH}_{2}\right) ; \quad 76.8(\mathrm{CH}) ; \quad 68.9\left(\mathrm{CH}_{2}\right) ; \quad 61.4\left(\mathrm{CH}_{3}\right) ;$ 60.1 $\left(\mathrm{CH}_{3}\right) ; 34.2\left(\mathrm{CH}_{2}\right)$ ppm. EI-MS $m / z 282[\mathrm{M}+\cdot]$.

13: white solid. ${ }^{1} \mathrm{H}$ NMR $\left(\mathrm{CDCl}_{3}\right): \delta 7.32(m, 5 \mathrm{H})$, $6.39(s, 1 \mathrm{H}), 5.89(s, 2 \mathrm{H}), 4.55(s, 2 \mathrm{H}), 4.02(s, 3 \mathrm{H})$, $3.99(m, 1 \mathrm{H}), 3.77(s, 3 \mathrm{H}), 3.49(d d, \mathrm{~J}=4.0,9.5 \mathrm{~Hz}$, $1 \mathrm{H}), 3.41(d d, \mathrm{~J}=7.0,9.5 \mathrm{~Hz}, 1 \mathrm{H}), 2.74(d, \mathrm{~J}=7.0 \mathrm{~Hz}$, 2H), $1.68(s, 1 \mathrm{H}) \mathrm{ppm} .{ }^{13} \mathrm{C}$ NMR/DEPT $\left(\mathrm{CDCl}_{3}\right): \delta$ 144.9(C), 144.8(C), 138.3(C), 137.8(C), 136.5(C), $127.9(\mathrm{CH}), \quad 129.0(\mathrm{CH}), \quad 124.1(\mathrm{C}), \quad 103.8(\mathrm{CH})$, $101.4\left(\mathrm{CH}_{2}\right), \quad 74.0\left(\mathrm{CH}_{2}\right), \quad 73.6\left(\mathrm{CH}_{2}\right), \quad 71.2(\mathrm{CH})$, 61.3 $\left(\mathrm{CH}_{3}\right), 60.1\left(\mathrm{CH}_{3}\right), 34.5\left(\mathrm{CH}_{2}\right)$ ppm. EI-MS: $\mathrm{m} / \mathrm{z}$ $346[\mathrm{M}+\cdot]$.

14: white solid. ${ }^{1} \mathrm{H}$ NMR $\left(\mathrm{CDCl}_{3}\right): \delta 8.03(d d, J=1.3$, $8.0 \mathrm{~Hz}, 1 \mathrm{H}), 7.61(t, J=8.0 \mathrm{~Hz}, 2 \mathrm{H}), 7.49(t, J=8.0 \mathrm{~Hz}$, $2 \mathrm{H}), 6.45(s, 1 \mathrm{H}), 5.88(s, 2 \mathrm{H}), 4.29(d d, J=4.5,11.0$ $\mathrm{Hz}, 1 \mathrm{H}), 4.22(d d, J=6.0,11.0 \mathrm{~Hz}, 1 \mathrm{H}), 4.14(m, 1 \mathrm{H})$, $3.97(s, 3 \mathrm{H}), 3.76(s, 3 \mathrm{H}), 2.88(d d, J=6.0,13.5 \mathrm{~Hz}$,
$1 \mathrm{H}), 2.78(d d, J=7.0,13.5 \mathrm{~Hz}, 1 \mathrm{H}), 1.55(s, 1 \mathrm{H}) \mathrm{ppm}$; ${ }^{13} \mathrm{C}$ NMR/DEPT $\left(\mathrm{CDCl}_{3}\right): \delta 166.8(\mathrm{C}), 144.9(\mathrm{C})$, 144.8(C), 137.7(C), 136.7(C), 133.0(CH), 132.9(CH), $129.7(\mathrm{C}), \quad 129.4(\mathrm{CH}), \quad 129.2(\mathrm{CH}), \quad 128.3(\mathrm{CH})$, 123.9(C), $\quad 103.6(\mathrm{CH}), \quad 101.4\left(\mathrm{CH}_{2}\right), \quad 69.8(\mathrm{CH})$, 68.1 $\left(\mathrm{CH}_{2}\right), 60.4\left(\mathrm{CH}_{3}\right), \quad 59.2\left(\mathrm{CH}_{3}\right), 34.6\left(\mathrm{CH}_{2}\right)$ ppm. EI-MS: $m / z 360[\mathrm{M}+\cdot]$.

\section{Acknowledgements}

The authors recognize financial supports for this research through grants from FAPEAM (PIPT 006/2006), FAPEAM (PPP 3007/2010) Brazilian Malaria Network (CNPq 561559/ 2008-2), BIONORTE Post-graduate Network (CNPq/2009) and FAPEAM Ph.D. scholarship and PCI-CNPq-INPA Research Fellowship.

\section{References}

Alibhai N., 1999, Part A: The Syntheses of dillapiol and its 4-thio derivatives. Part B: The synthesis of trichiliasterone B, Master's Dissertation, University of Ottawa, Canada, pp.123

Amiguet V.T., Petit P., Ta C.A., Nuñez R., Sánchez-Vindas P., Alvarez L.P., Smith M.L., Arnason J.T., and Durst T., 2006, Phytochemistry and antifungal properties of the newly discovered tree Pleodendron costaricence, J. Nat. Prod., 69: 1005-1009 http://dx.doi.org/10.1021/np0504863

Barreto C.F., 2005, Aedes aegytpi - Resistência aos inseticidas químicos e as novas alternativas de controle, Rev. Eletrônica, Faculdade Montes Belos, 1: 62-73

Belzile A.S., Majerus S.L., Podeszfinski C., Guillet G., Durst T., and Arnason J.T., 2000, Dillapiol derivatives as synergists: structure-activity relationship analysis, Pestic. Biochem. Phys., 66: 33-40 http://dx.doi.org/10.1006/pest.1999.2453

Bernard C.B., Arnason J.T., Philogène, B.J.R., Lam J., and Waddell T., 1989, Effect of lignans and other secondary metabolites of the Asteraceae on the mono-oxygenase activity of the European corn borer, Phytochemistry, 28: 1373-1377

http://dx.doi.org/10.1016/S0031-9422(00)97749-5

Bernard C.B., Krishnamurty H.G., Chauret D., Durst T., Philogène B.J.R., Sánchez-Vindas P., Hasbun C., Poveda L., San R.L. and Arnason J.T., 1995, Insecticidal defenses of Piperaceae from the neotropics, J. Chem. Ecol., 21: 801-815 http://dx.doi.org/10.1007/BF02033462

Braga I.A., and Valle D., 2007, Aedes aegypti: inseticidas, mecanismo de ação e resistência, Epidemiol. Serv. Saude, 16: 279-293

Campos J., and Andrade C.F.S., 2001, Susceptibilidade larval de duas populações de Aedes aegypti a inseticidas químicos, Rev. Saude Publica, 35: 232-236 http://dx.doi.org/10.1590/S0034-89102001000300003

Cassida J.E., 1970, Mixed-function oxidase involvement in the biochemistry of insecticide synergists, J. Agri. Food Chem., 18: 753-772 http://dx.doi.org/10.1021/jf60171a013 
Cicció J.F., and Ballestero C.M., 1997, Constituyentes volátiles de las hojas y espigas de Piper aduncum (Piperaceae) de Costa Rica, Rev. Biol. Trop., 45: 783-790

Conover W.J., eds., 1999, Practical Nonparametric Statistics, 3rd edition, John Wiley and Sons, New York, pp.584

Chapagain B.P., Saharan V., and Wiesman Z., 2008, Larvicidal activity of saponins from Balanites aegyptiaca callus against Aedes aegypti mosquito, Bioresource Technol., 99: 1165-1168 http://dx.doi.org/10.1016/j.biortech.2007.02.023

Demetzos C., Perdetzoglou D., Gazouli M., Tan K., and Economakis C., 2000, Chemical analysis and antimicrobial studies on three species of Ferulago from Greece, Planta Med., 66: 560-563 http://dx.doi.org/10.1055/s-2000-8652

Georges K., Jayaprakasam B., Dalavoy S.S., and Nair M.G., 2008, Pest-managing activities of plant extracts and anthraquinones from Cassia nigricans from Burkina Faso, Bioresource Technol., 99: 2037-2045 http://dx.doi.org/10.1016/j.biortech.2007.02.049

Gomes E.O., Tadei W.P., and Nunomura S.M., 2005, Estudo da família Piperaceae quanto a atividade larvicida e adulticida, contra Aedes aegypti (Linnaeus, 1762), Rev. Soc. Bras. Med. Trop., 38: 50-51

Gomes E.O., Tadei W.P., and Nunomura S.M., 2006, As potencialidades do dilapiol como agente sinergístico no controle da malária e dengue, Rev. Soc. Bras. Med. Trop., 39: 201

Larocque N., Vincent C., Bélanger A., and Bourassa J.P., 1999, Effects of tansy essential oil from Tanacetum vulgare on biology of oblique-banded leafroller, Choristoneura rosaceana, J. Chem. Ecol., 25: 1319-1330 http://dx.doi.org/10.1023/A:1020974725437

Lichtenstein E.P., Liang T.T., Schulz K.R., Schnoes H.K., and Carter G.T., 1974, Insecticidal and synergistic components isolated from dill plants, J. Agr. Food Chem., 22: 658-664 http://dx.doi.org/10.1021/jf60194a037

Luna J.E.D., Martins M.F., Dos Anjos A.F., Kuwabara E.F., and Navarro-Silva M.A., 2004, Susceptibilidade de Aedes aegypti aos inseticidas temephos e cipermetrina, Brasil, Rev Saúde Pública, 38: 842-843 http://dx.doi.org/10.1590/S0034-89102004000600013

Majerus S.L., 1997, A novel synthesis of dillapiol amd its derivatives and their role as chemosensitizes and insecticide synergists, Thesis for M.S., Universite of Otawa, Canada, pp.133

Mukerjee S.K., Saxena V.S., and Tomar S.S., 1979, New methylenedioxyphenyl synergists for pyrethrins, J. Agr. Food Chem., 27: 1209-1211 http://dx.doi.org/10.1021/jf60226a033
Nerio L.S., Olivero-Verbel J., and Stashenko E., 2010, Repellent activity of essential oils: A review, Bioresource Technol., 101: 372-378 http://dx.doi.org/10.1016/j.biortech.2009.07.048

Omar S., Godard K., Ingham A., Hussain H., Wongpanich V., Pezzuto J., Durst T., Eklu C., Gbeassor M., Sanchez-Vindas P., Poveda L., Philogene B.J.R., and Arnason, J.T., 2003, Antimalarial activities of gedunin and 7-methoxygedunin and synergistic activity with dillapiole, Ann. Appl. Biol., 143: 135-141 http://dx.doi.org/10.1111/j.1744-7348.2003.tb00279.x

Pinto, A.C.S., 2008, Desenvolvimento de Substâncias Semi-Sintétícas e Bioativas a partir de 4-Nerolidilcatechol e Dilapiol, Ph.D. Thesis, University of Amazonas, pp.298

Pohlit A.M., Pinto A.C.S., Cavalcanti B.C., Pessoa C.O., Silva E.C.C., Chaves F.C.M., Nogueira K.L., Costa-Lotufo L.V., Silva L.F.R., Melo M.R.S., Vieira P.P.R., Mause R., Amorim R.C.N., Tadei W.P., and Andrade Neto V.F., 2008, Biopharmaceuticals Synthesis. In: Carioca J.O.B., organizer, Brazilian Network on Green Chemistry- Awareness, Responsability and Action, Fortaleza-CE: Edições UFC, pp.347-372

Pohlit A.M., Lopes N.P., Gama R.A., Tadei W.P., and Andrade Neto V.F., 2011a, Patent literature on mosquito repellent inventions which contain plant essential oils - a review, Planta Med., 77(6): 598-617 http://dx.doi.org/10.1055/s-0030-1270723

Pohlit A.M., Baldin E.L.L., Rezende A.R., Lopes N.P., and Andrade Neto V.F., 2011b, Plant extracts, isolated phytochemicals, and plant-derived agents which are lethal to arthropod vectors of human tropical diseases - a review, Planta Med., 77(6): 618-630 http://dx.doi.org/10.1055/s-0030-1270949

Shaalan E.A.S., Canyon D., Younes M.W.F., Abdel-Wahab H., and Mansour A.H., 2005, A review of botanical phytochemicals with mosquitocidal potential, Environ. Int., 31: 1149-1166 http://dx.doi.org/10.1016/j.envint.2005.03.003

Tomar S.S., Maheshwari M.L., and Mukerjee S.K., 1979a, Synthesis and synergistic activity of some pyrethrum synergists from dillapiole, Agr. Biol. Chem., 43: 1479-1483 http://dx.doi.org/10.1271/bbb1961.43.1479

Tomar S.S., Maheshwari M.L., and Mukerjee S.K., 1979b, Synthesis and synergistic activity of dillapiole based pyrethrum synergists, J. Agr. Food Chem., 27: 547-550 http://dx.doi.org/10.1021/jf60223a012

WHO, 2006, Guidelines for testing mosquito adulticides for indoor residual spraying and treatment for mosquito nets. 6a. edition, 1: 114 